\title{
Article
}

\section{Review on Dark Matter Searches with Neutrino Telescopes}

\author{
Juan de Dios Zornoza ${ }^{1,+}$ (†000-0002-1834-0690*
}

1 IFIC - Instituto de Física Corpuscular (UV-CSIC),

c/ Catedrático José Beltrán, 2. CP 46980, Valencia (Spain);

\begin{abstract}
The search for dark matter is one the hottest topics in Physics today. The fact that about $80 \%$ of the matter of the Universe is of unknown nature has triggered an intense experimental activity to detect such kind of matter and a no less intense effort on the theory side to explain it. Given the fact that we do not know well the properties of dark matter, searches from different fronts are mandatory. Neutrino telescopes are part of this experimental quest and offer specific advantages. Among the targets to look for dark matter, the Sun and the Galactic Center are the most promising ones. Neutrino telescopes have put the best limits on spin-dependent cross section of proton-WIMP scattering. Moreover, they are competitive in the constraints on the thermally averaged annihilation cross-section for high WIMP masses when looking at the Galactic Centre. Other results are also reviewed.
\end{abstract}

Keywords: dark matter; neutrino telescopes, IceCube, ANTARES, KM3NeT, SuperK

Citation: Zornoza, J.D. Review on Dark Matter Searches with Neutrino Telescopes. Preprints 2021, 1, 0. https://doi.org/

Received:

Accepted:

Published:

Publisher's Note: MDPI stays neutral with regard to jurisdictional claims in published maps and institutional affiliations.

\section{Introduction}

During several decades we have been gathering overwhelming experimental evidence of the existence of new kind of matter, dubbed "dark matter", which constitutes more than $80 \%$ of the matter content of the Universe but whose nature is unknown [1,2]. We have proofs of its gravitational effects, but it seems that it does not interact with light. We also know that is should be stable, or at least stable enough to have survived until our days. There is no candidate in the Standard Model whose properties fits with the properties inferred for this kind of matter, so many alternative theories implying New Physics have have been proposed. Some of these models propose changes in the gravitation theory [3], instead of assuming the existence of a new kind of particles, although in this review we will focus on the first scenario.

Since we only partially know the properties of this new kind of matter, it is not possible to assure which is the best experimental strategy for its detection. Therefore, a multi-front attack is needed, which includes direct detection experiments, colliders and indirect searches. Indirect searches use gamma rays, cosmic rays and cosmic neutrinos (see [4] for a general review). In this review, we will focus on the experimental basis and results of the searches with neutrino telescopes. Previous reviews on this topic can be found in $[5,6]$. As it will be seen, neutrino telescopes have their own specific advantages and complement other searches.

The structure of this review is as follows. In Section 2 we will describe the detection principle and the main experiments (IceCube, ANTARES, KM3NeT, SuperK). Then, in Section 3 we will review the experimental results obtained for the most promising sources (Sun, Earth, Galactic Centre, galaxy clusters and dwarf galaxies). For the best candidates (Sun and Galactic Centre), a brief digression on the phenomenology will be included. Finally, we will summarize the main conclusions and discuss the prospects for the future in Section 4.

\section{Materials and methods}

The basic design of neutrino telescopes was proposed by M. Markov [7] in 1961. The idea is to install light detectors at large depth in the sea, lakes or the Antarctic ice (i.e. large volumes of transparent target) in order to detect the Cherenkov light induced by charged leptons produced in the interaction of high energy neutrinos with the matter 
in/surrounding the instrumented volume. Two main kinds of topologies are possible. On the one hand, tracks produced by muons originated mainly in the charged-current interaction of muon neutrinos. On the other hand, showers are produced after the neutralcurrent interactions of neutrinos of any flavour or after the charged-current interactions of electron and tau neutrinos. The former allow for a better angular resolution, since they are long tracks which can reach hundreds of meters (depending on the energy), while showers are seen as bright spheres given the granularity of the light sensors in these detectors.

The main background for neutrino telescopes comes from the interaction of cosmic rays in the atmosphere. These interactions produce a huge flux of down-going muons, which severely limits the capacity of these detectors to look for down-going events. Moreover, an almost isotropic background of atmospheric neutrinos is also produced by these interactions, which is dominant among up-going events [8].

As it will be described later in this review, neutrino telescopes can be used to look for dark matter since production of neutrinos is expected (typically as secondaries, but in principle also possible as primary yield) after the annihilation or decay of dark matter particles. A detailed discussion about production mechanisms can be found in [9]. An excess of high energy neutrinos in the direction of the sources of interest would be a hint of dark matter accumulation.

- IceCube The IceCube Neutrino Observatory [10] is located at the South Pole. Its instrumented volume is one cubic kilometre, being the largest neutrino telescope to date. It consists of 86 strings, about one kilometre length (from 1450 to $2450 \mathrm{~m}$ deep), equipped with 60 Digital Optical Modules (DOMs) each. A DOM contains a 10 " photomultiplier (PMT) and the associated electronics for signal processing. It was completed in 2010. As it happens with other detectors of this kind, data taking and physics analyses can start as soon as a few lines are installed. The observatory also includes a surface array for cosmic rays, called IceTop [11], which can be used as a veto. Recently, an upgrade with six more lines was added in the internal part of the detector. There are on-going plans for the expansion of the observatory to a larger scale and additional subdetectors [12].

- $\quad$ ANTARES The ANTARES telescope [13] is installed in the Mediterranean Sea, close to the French coast, at a depth of $1450 \mathrm{~m}$. It consists of 12 lines, with a length of $450 \mathrm{~m}$, with 75 Optical Modules (OMs) each (except for one of the lines, partly instrumented with acoustic detectors. It was completed in 2008 and its decommissioning is planned for 2021. Although smaller than IceCube, it offers a better angular resolution, due to the longer scattering length of light in water, and a better visibility of the Galaxy (including the Galactic Center, particularly important for some of the searches discussed here).

- $\quad$ KM3NeT The KM3NeT Observatory [14] is being constructed in the Mediterranean Sea, in two different locations. The ORCA subdetector is being installed in front of the French coast, at a depth of $2500 \mathrm{~m}$, not far from the ANTARES site, while the ARCA subdetector is being installed off the Sicilian coast at a depth of $3500 \mathrm{~m}$. ORCA, with 115 lines with 60 DOMs each, is denser and smaller (8 MTon), with a low energy threshold (about $3 \mathrm{GeV}$ ). ARCA, with 230 lines (longer but also equipped with 60 DOMs each), will have a total volume of about one cubic kilometer. DOMs in KM3NeT are equipped with 31 3-inch PMTs. At the time of this writing, 6 lines of ORCA and 8 lines of ARCA are already installed and taking data, with more lines to be imminently deployed.

- SuperK SuperK [15] is a different concept of detector, although with some similarities with the ones mentioned above. Installed in a mine in Kamioka (Japan), the detector consisted in a pool of purified water ( $22 \mathrm{kT}$ of fiducial volume) surrounded by PMTs. Its data have been used to look for neutrinos from the Sun, in an energy regime lower than the other detectors described in this review. Recently, Gadolinium has been added to the tank for its optimization for supernova detection. 


\section{Results}

A popular general candidate explaining dark matter are WIMPs (Weakly Interacting Massive Particles), being the neutralino one of the most studied realisation of this possibility, since it can be the lightest super-symmetry particle in SuperSymmetry models [16].

The search for dark matter with neutrino telescopes can be performed for several sources. We can distinguish between two different scenarios, though, as we will discuss later. For sources like the Sun or the Earth, we expect WIMPs present in the Solar System to scatter off nucleons of the Sun or the Earth. These dark matter particles would lose energy and eventually would become gravitationally trapped. Their annihilation with other dark matter particles (if they are assumed to be Majorana particles) would produce (directly or indirectly) high-energy neutrinos. For other sources (Galactic Centre, galaxy clusters, dwarf galaxies...) we would rather expect dark matter particles to have accumulated during the structure formation in these sources just by gravitational attraction. Again, the encounters among these particles would produce neutrinos after their annihilation. It is also possible that dark matter is not stable (although stability at the scale of the age of the Universe is required to ensure its present existence) and decay producing neutrinos.

In the following, we will review recent results for the sources mentioned above.

\subsection{Sun}

The Sun is one of the most promising targets for dark matter searches with neutrino telescopes. As mentioned earlier, dark matter particles in the Solar System are expected to occasionally scatter off protons (and neutrons) in the Sun. They would lose energy and become gravitationally trapped. As the density of dark matter particles increases with time, self-annihilation of these particles also increases. At the scales of the Sun's lifetime, the density will be high enough to reach equilibrium between capture and annihilation.

If we can assume that capture rate and annihilation cross section are large, we can expect that equilibrium has been reached. The rate of change of the number of WIMPs $N(t)$ in the Sun would be [17]

$$
\dot{N}(t)=C^{\odot}-A^{\odot} N(t)^{2}-E^{\odot} N,
$$

where $C^{\odot}$ is the capture rate, $A^{\odot}$ is the annihilation cross-section times the relative WIMP velocity per volume and $E^{\odot}$ is the evaporation term, i.e. the inverse time for a WIMP to escape (for those with in the tail of large velocities). The evaporation term can be neglected for WIMPs heavier than a few $\mathrm{GeV}$ [18].

For the Sun, it can be assumed that equilibrium between capture and annihilation has been reached $\left(\sqrt{C^{\odot} A} t_{\odot} \gg 1\right)$, being $t_{\odot} \approx 4.5 \times 10^{9}$ years [17]:

$$
\Gamma=\frac{1}{2} A^{\odot} N\left(t_{\odot}\right)^{2}=\frac{1}{2} C^{\odot} \tanh ^{2}\left(\sqrt{C^{\odot} A \odot} t_{\odot}\right) .
$$

Therefore, for this case we can set a relationship between the annihilation rate (on which we can set an estimation or a limit from the observation/non-observation of events in our neutrino telescope) and the scattering cross section (since this is related to the capture rate).

Due to the relationship explained above, the searches for neutrino telescopes for dark matter in the Sun set limits in the scattering cross section, as direct searches do. In this sense, neutrino telescopes can be seen as direct search experiments where the Sun is the target. Interestingly, direct searches and neutrino telescopes searches are complementary. Neutrino telescopes are more sensitive to the part of lower velocities of the WIMP velocity distribution (since those WIMPs are more easily captured), while direct searches are more sensitive to the part of higher velocities (since these WIMPs will produce larger signals in the detectors).

Figure 1 shows recent results of several experiments. As mentioned above, these results can be compared with direct search experiments. It can be seen that for the spindependent part of the cross-section, neutrino telescopes (and IceCube in particular), offer 
the best present limits. This particular effectiveness of neutrino telescopes for the spindependent part comes from the the fact that the Sun is made mainly of protons. The best region for SuperK is at low masses, given its low energy threshold, while for ANTARES, the contrary happens, being much larger than SuperK.
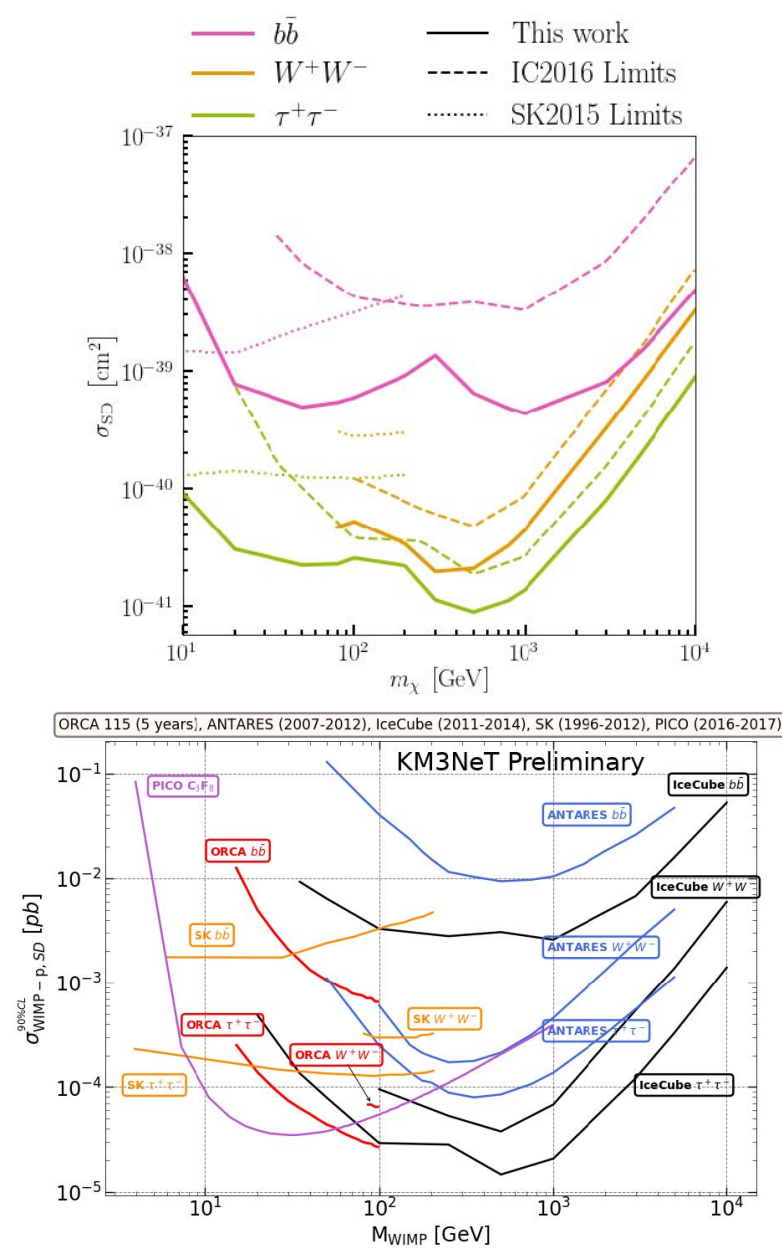

Figure 1. Limits on the spin-dependent WIMP-proton scattering cross section as a function of the WIMP mass for several channels: (top) Limits from IceCube [19,20] and SuperK [21] (figure reproduced from [19], credit: IceCube collaboration); (bottom) Limits from ANTARES [22] and sensitivity (for five years) for KM3NeT-ORCA [23]; limits from IceCube (3 years of data) [24], SuperK [21] and PICO [25] are also shown (figure reproduced from [23], credit: ANTARES and KM3NeT collaborations).

It should be mentioned that an important advantage of dark matter searches in the Sun by neutrino telescopes is the fact that the astrophysical backgrounds are small, compared to other indirect searches. The only relevant background at these energies would be from the interaction of cosmic rays with the Sun's corona [26,27], the so-called atmospheric solar neutrinos. Therefore, a potential excess of high energy neutrinos from the Sun would be a very clean signal of dark matter (in opposition to searches with gamma rays, cosmic rays, etc., for which the uncertainties in astrophysical backgrounds are larger).

Other interesting searches in the Sun include those looking for secluded dark matter. In these models, dark matter particles can be coupled to Standard Model particles through long lived mediators than can escape from the Sun and decay into SM particles, including muons and neutrinos, which can produce signals in neutrino telescopes. Results for these models for the Sun can be found in [28-30]. 


\subsection{Earth}

The process for dark matter accumulation in the Earth is similar to what happens in the Sun, with some relevant differences. First, it cannot be assumed that equilibrium between capture and annihilation has been reached at the Earth given its smaller size, compared to the Sun and also to the influence of the latter in the capture process at the Earth.

Moreover, the scattering of dark matter particles occurs mainly with spin-zero nuclei like ${ }^{56} \mathrm{Fe},{ }^{28} \mathrm{Si}$ and ${ }^{16} \mathrm{O}$ [4]. Therefore, limits for this case are particularly good for spin-independent scattering cross-section, since it benefits from the dependence of the interaction cross section on the squared atomic number of the target.

Figure 2 shows limits obtained by ANTARES and SuperK and sensitivity of IceCube for dark matter searches in the Earth. Because of kinematics, limits and sensitivities are particularly good for the WIMP masses close to those of the most abundant nuclei.

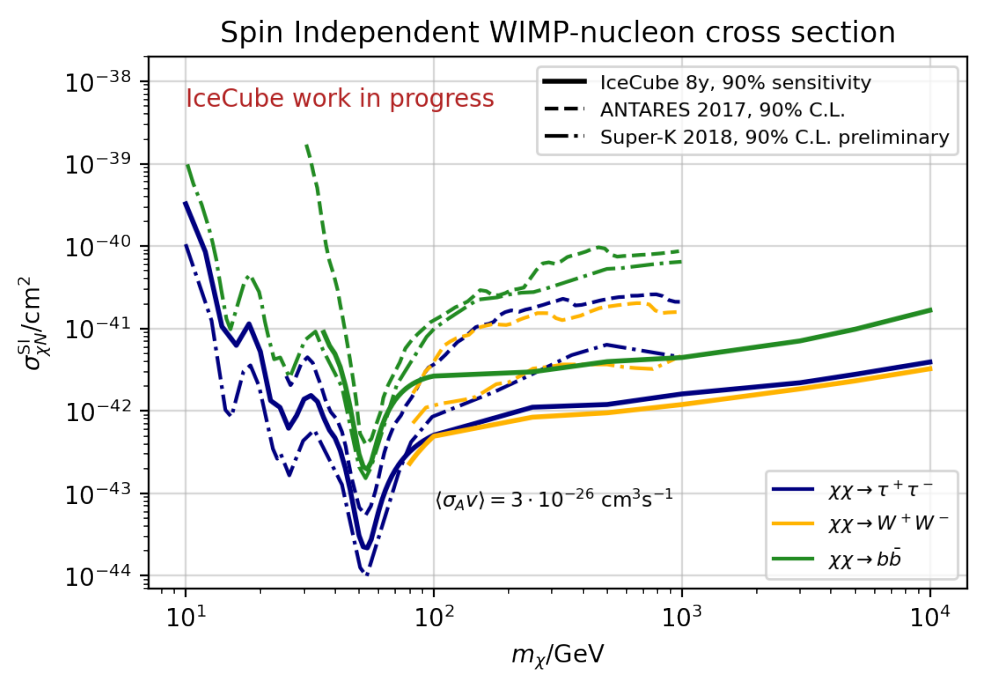

Figure 2. Limits on the spin-independent WIMP-nucleon scattering cross-section of ANTARES [31], SuperK [32] and sensitivities of IceCube [33] at 90\% C.L. Figure reproduced from [33], credit: IceCube collaboration.

\subsection{Galactic Centre}

The Galactic Center is an excellent place to look for dark matter using neutrino telescopes. As mentioned above, signals come from the annihilation (or decay) of dark matter particles into neutrinos (directly or, more likely, after the decay of secondaries from those annihilations). Limits are therefore set on the thermally averaged annihilation cross section. Other messengers, like gamma rays, would also be produced in these processes, but the corresponding searches suffer from large astrophysical backgrounds, so they are focused in other sources like dwarf galaxies.

The measured flux (or limit on it) can be related to the annihilation cross-section as follows [34]:

$$
\frac{d \phi_{v}}{d E_{v}}=\frac{1}{4 \pi} \frac{\left\langle\sigma_{A} v\right\rangle}{2 m_{D M}^{2}} \frac{d N_{v}}{d E_{v}} J_{\Psi}
$$

where $m_{D M}$ is dark matter particle mass and $d N_{v} / d E_{v}$ is the differential number of neutrinos produced per annihilating pair of dark matter particles. Typically a $100 \%$ branching ratio to a given channel of interest is assumed. Among these channels, $b \bar{b}$ would represent a soft-spectrum case, while $\tau \bar{\tau}$ would give a hard spectrum. The so-called J-factor, $J_{\Psi}$ is defined as

$$
J_{\Psi}=\int_{\Delta \Omega} d \Omega(\Psi) \int_{l . o . s .} \rho_{D M}^{2}(r(l, \Psi)) d l,
$$


where $r$ is the distance to the Galactic Centre; $\Delta \Omega$, the solid angle, and $\rho_{D M}$, the dark matter density. Examples of dark matter halo profiles are given by Burkert [35] and by Navarro-Frenk-White [36].

Recent results of searches in the Galactic Centre are shown in Figure 3, where limits to the thermally averaged annihilation cross section are plotted as a function of the WIMP mass. ANTARES, although much smaller than IceCube, has the advantage of being installed in the Northern Hemisphere, which allows for a better visibility of the Galactic Centre. This explains why ANTARES limits are orders of magnitude better than those of IceCube for large dark matter masses. As it can be seen, the annihilation cross section can also be probed by other indirect searches. Particularly constraining are the limits in the low DM mass region from Fermi-LAT looking at dwarf spheroidals and HESS in the medium mass region looking at the Galactic Centre. It is worthy to note that the results from ANTARES are particularly competitive in the region of very large dark matter mass. In this region, mechanisms to avoid the limits from unitarity [37] have to be claimed (see examples in [9]), but are becoming increasing interesting given the null results from other searches at lower masses, in particular from colliders. 

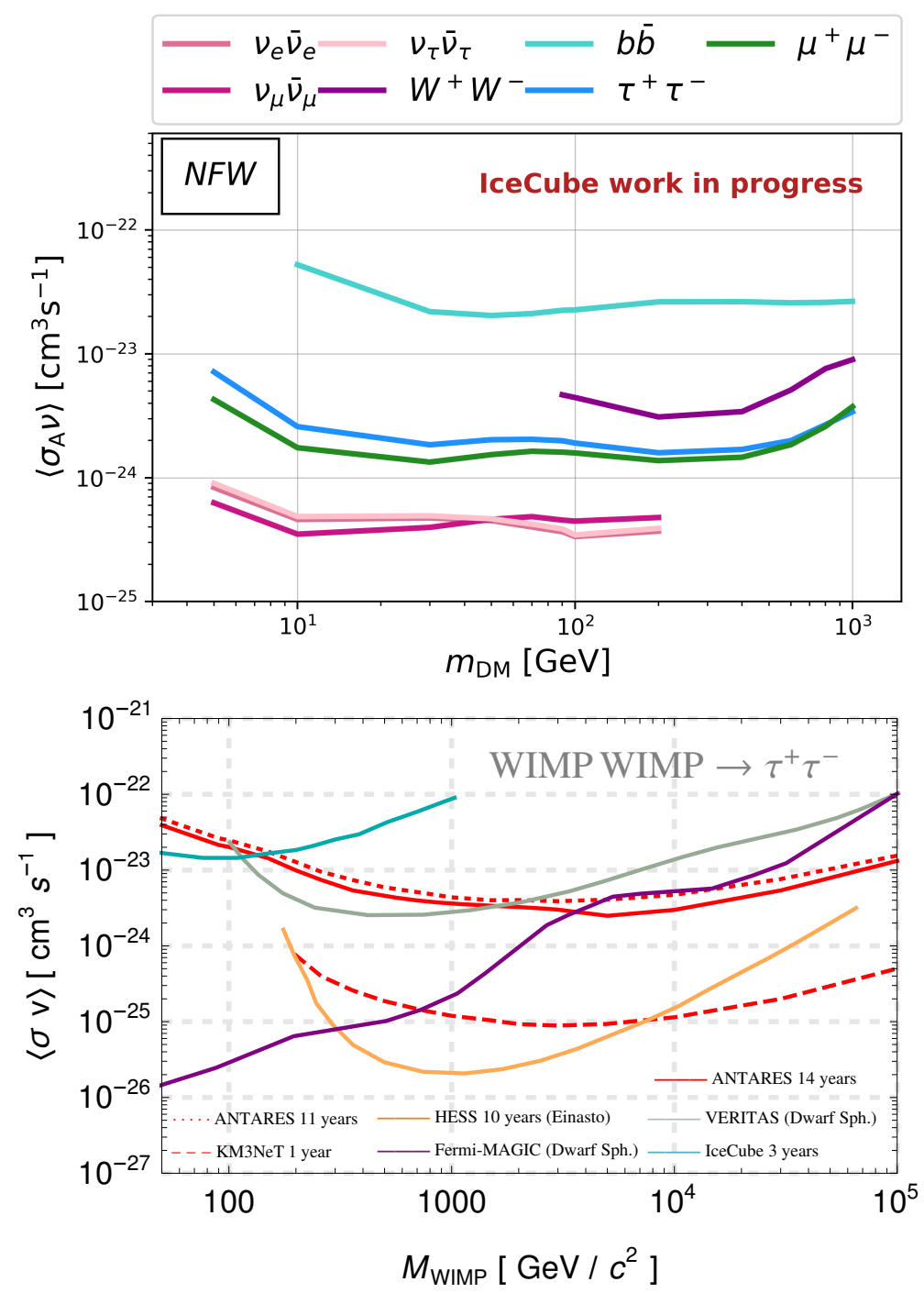

Figure 3. Upper limits at $90 \%$ C.L. on the thermally averaged cross-section for WIMP annihilation as a function of the WIMP candidate mass. top Limits from IceCube [38] for several channels; credit: IceCube collaboration; bottom Limits from ANTARES [39] and sensitivity for KM3NeT-ARCA (one year) [40], compared to other results (IceCube 2017 [41], HESS [42], VERITAS [43] and FermiLAT+MAGIC [44]) for the $\tau^{+} \tau^{-}$channel and using the NFW halo profile [36] unless indicated otherwise. Figure reproduced from [40]; credit: ANTARES and KM3NeT collaborations.

It is also worth mentioning the analysis carried out jointly by the IceCube and ANTARES collaborations, combining data from both experiments [45]. This allows to improve by a factor two the limits in the region where the sensitivities from both experiments are comparable. Moreover, it represented also an opportunity to harmonize analysis tools from both collaborations.

Finally, we would like to mention that sensitivities for secluded dark matter scenarios in the Galactic Center also exist [46].

\subsection{Other sources}

Among other possible sources to look for dark matter with neutrino telescopes we can mention the following: the Galactic Halo, nearby dwarf galaxies and galaxy clusters. Particularly interesting results from these sources come from the searches for dark matter decay. As mentioned earlier, the decay lifetimes should be large enough compared to the age of the Universe so that dark matter has survived in the amounts observed nowadays. Figure 4 summarizes some of these analyses, compared to the limits set by other indirect 
searches. As it can be seen, they can provide very competitive limits on the life time of heavy dark matter particles.

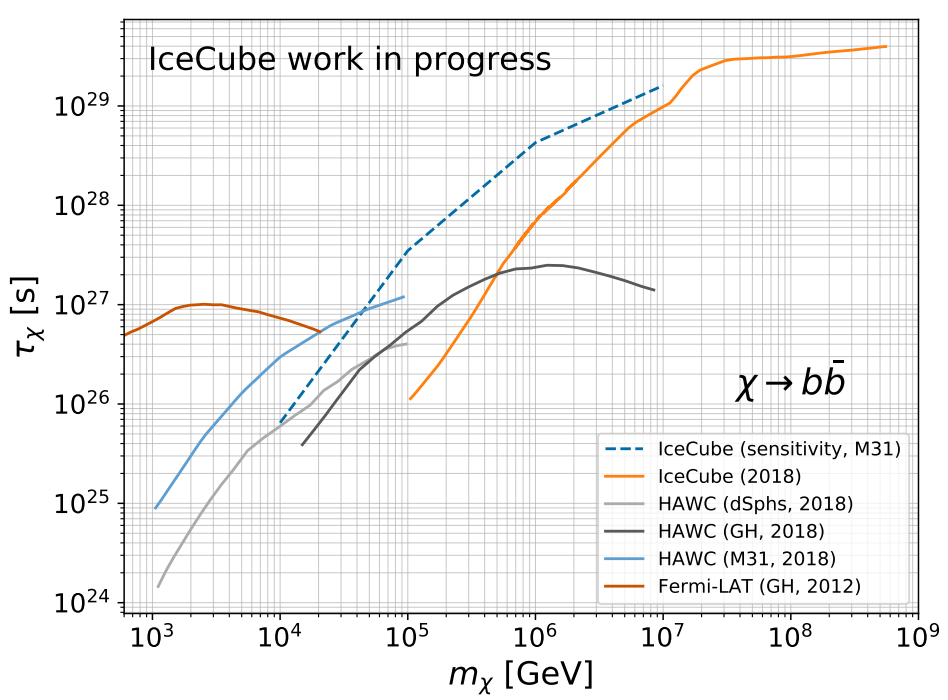

Figure 4. Limits and sensitivity on decaying dark matter life time from different experiments [47-51]. Figure reproduced from [52], credit: IceCube collaboration.

\section{Discussion and conclusions}

No signal of dark matter has been found yet by neutrino telescopes. As we have seen, these detector have specific advantages and have provided competitive limits in some relevant case. For searches in the Sun, astrophysical background is small and well understood. IceCube has set the most stringent limits in the world for spin dependent cross section of nucleon-WIMP scattering. In the Galactic Centre, ANTARES has the advantage of a better visibility of this regions, which translates into better limits than IceCube for medium and large masses. This region of large masses in becoming more interesting as searches at lower masses are coming hands empty. For models of decaying dark matter, IceCube has also constrained the heavy dark matter lifetime beyond other searches.

In the close future, neutrino telescopes under construction like KM3NeT, HyperK [53], Baikal-GVD [54] will push further these limits, maybe up to the point of discovery. Estimations of the sensitivity of KM3NeT are shown in Figure 1 and 3. In any case and wherever the first dark matter signal comes from, it will be necessary to study the possible candidates with from all the experimental fronts. For this, neutrino telescopes will bring the complementary view discussed in this review.

Funding: The author acknowledges the financial of support of the Ministerio de Ciencia, Innovación, Investigación y Universidades (MCIU): Programa Estatal de Generación de Conocimiento (ref. PGC2018-096663-B-C41) (MCIU/FEDER).

Conflicts of Interest: The author is member of the ANTARES and KM3NeT collaborations.

\section{References}

1. Bertone, G and Hooper D. History of dark matter. Rev. Mod. Phys. 2018, 90, 4, 045002.

2. Bertone, G. and Silk J. Particle dark matter. In Particle dark matter; Bertone G., Eds.; University Press: Cambridge, United Kingdom, 2010; pp. 3-13.

3. Famaey, B.; McGaugh, S. Modified Newtonian Dynamics (MOND): Observational Phenomenology and Relativistic Extensions. Living Rev. Rel. 2012, 15, 10.

4. Pérez-de-los-Heros, C. Status, Challenges and Directions in Indirect Dark Matter Searches Symmetry 2020, 12, 1648. 
5. Zornoza, J.D. Indirect search for dark matter with neutrino telescopes. In Proceedings of the 14th Marcel Grossmann Meeting on Recent Developments in Theoretical and Experimental General Relativity, Astrophysics, and Relativistic Field Theories (MG14), Rome, Italy, July 12-18, 2015; Bianchi M. , Jantzen R.T., Ruffini R., Eds.; 2017 pp. 3257-3261.

6. Pérez-de-los-Heros, C. The quest for dark matter with neutrino telescopes. In Neutrino Astronomy-Current status, future prospects; Gaisser T. and Karle A., Eds.; World Scientific: Singapur 2017; Chapter 11.

7. Markov M.A., On high energy neutrino physics. In: Proc. of 10th ICHEP, Rochester, 1960, p. 578.

8. Gaisser, T.K. The atmospheric neutrino flux. In Probing particle physics with neutrino telescopes; Pérez de los Heros, C, Eds.; World Scientific: Singapur 2020; Chapter 3.

9. Palomares-Ruiz, S. Tests of dark matter scenarios with neutrino telescopes. In Probing particle physics with neutrino telescopes; Pérez de los Heros, C, Eds.; World Scientific: Singapur 2020; Chapter 7.

10. Aartsen, M.G. et al (IceCube Collaboration) The IceCube Neutrino Observatory: Instrumentation and Online Systems JINST 2017, 12, 03 P03012.

11. Abassi, R. et al (IceCube Collaboration) IceTop: The surface component of IceCube, Nucl. Instrum. Meth. A 2013 700 188-220.

12. Aartsen, M.G. et al. (IceCube Collaboration) IceCube-Gen2: The Window to the Extreme Universe. arXiv 2020, arXiv:2008.04323

13. Ageron, M. et al. (ANTARES Collaboration), ANTARES: The first undersea neutrino telescope, Nucl. Inst.. and Meth. in Phys. Res. A 2011656 11-38.

14. Adrian-Martinez, S. et al. (KM3NeT Collaboration) Letter of intent for KM3NeT 2.0. J. Phys. G 2016, 43, 084001

15. Fukuda S. et al. (Super-Kamiokande Collaboration). The SuperKamiokande detector. Nucl. Instruments and Methods A 2003501 418

16. Jungman, G.; Kamionkowski, M.; Griest, K. Supersymmetric dark matter. Phys. Rept. 1996, $267,195$.

17. Halzen, F. and Hooper, D. High-energy neutrinos from WIMP annihilations in the Sun. In Particle dark matter; Bertone G., Eds.; University Press: Cambridge, United Kingdom, 2010; pp. 507-520.

18. Griest, K. and Seckel, D. Cosmic asymmetry, neutrinos and the Sun. Nuclear Physics B 1987 283, pp. 681-705.

19. Lazar, J. Solar WIMP Search with 10 Years of IceCube Data, Presentation at Very Large Volume Neutrino Telescope Workshop 2021, Valencia (Spain), Available online: https:/ indico.ific.uv.es/event/3965/contributions/14806/ (accessed on 28/09/2021)

20. Aartsen M.G. et al. (IceCube Collaboration), Improved limits on dark matter annihilation in the Sun with the 79-string IceCube detector and implications for supersymmetry, JCAP 2016 04, 022.

21. Choi, K. et al. (SuperKamiokande collaboration) Search for neutrinos from annihilation of captured low-mass dark matter particles in the Sun by Super-Kamiokande, Phys. Rev. Lett. 2015, 114, 141301.

22. Adrián-Martínez, S. et al. (ANTARES Collaboration), Limits on dark matter annihilation in the sun using the ANTARES neutrino telescope, Phys. Lett. B 2016 759. pp. 69-74.

23. Lopez-Coto, D. et al. Sensitivity of the ANTARES neutrino telescope for secluded dark matter searches. In Proceedings of the 36th International Cosmic Ray Conference (ICRC2019), Madison-WI, U.S.A., 2019; PoS(ICRC2019) 536.

24. Aartsen, M.G. et al. (IceCube collaboration), Search for annihilating dark matter in the Sun with 3 years of IceCube data, Eur.Phys.J. C 2017 77, no.3, 146

25. Amole, C, et al. (PICO Collaboration), Dark Matter Search Results from the PICO-60 C3F8 Bubble Chamber, Phys. Rev. Lett. 2017 118, no.25, 251301.

26. Aartsen M.G. et al. (IceCube Collaboration) Searches for neutrinos from cosmic-ray interactions in the Sun using seven years of IceCube data. JCAP 202102025.

27. López-Coto, D. et al. Solar Atmospheric Neutrinos searches with ANTARES neutrino telescope. In Proceedings of the 37th International Cosmic Ray Conference (ICRC2021), Berlin, Germany, 2021; PoS(ICRC2021) 122 pp. 1122.

28. Adrian-Martinez, S. et al. (ANTARES Collaboration) Results of the search for Secluded Dark Matter in the Sun with the ANTARES neutrino telescope,JCAP 20161605 05, 016,

29. Ardid, M. et al. Constraining Secluded Dark Matter models with the public data from the 79-string IceCube search for dark matter in the Sun, JCAP 2017 04, 010.

30. Toennis, C. et al. Search for secluded dark matter with 6 years of IceCube data. In Proceedings of the 37th International Cosmic Ray Conference (ICRC2021), Berlin, Germany, 2021; PoS(ICRC2021) 521.

31. Albert, A. et al. (ANTARES Collaboration) Search for dark matter annihilation in the earth using the ANTARES neutrino telescope, Phys. Dark Univ. 201716 pp. 41-48.

32. Mijakowski, P. et al. (Super-Kamiokande Collaboration), Journal of Physics: Conference Series 20201342012075.

33. Renzi, G. et al. Search for dark matter from the center of the Earth with 8 years of IceCube data. In Proceedings of the 37th International Cosmic Ray Conference (ICRC2021), Berlin, Germany, 2021; PoS(ICRC2021) 526.

34. Yuksel, H. et al. Neutrino constraints on the dark matter total annihilation cross section Phys. Rev D 2007, 76, 123506.

35. Burkert, A. Searching the Parameters of Dark Matter Halos on the Basis of Dwarf Galaxies' Dynamics Astrophys. J. Lett. 1995, 447, L25.

36. Navarro, J.F. et al. The Structure of Cold Dark Matter Halos Astrophys. J. 1996, 462, 563-575.

37. Blum, K.; Cui, Y.; Kamionkowski, M. An Ultimate Target for Dark Matter Searches. Phys. Rev. D 2015, 92, 023528.

38. Iovine, N. et al. Indirect search for dark matter in the Galactic Centre with IceCube. In Proceedings of the 37th International Cosmic Ray Conference (ICRC2021), Berlin, Germany, 2021; PoS(ICRC2021) 524. 
39. Albert A. et al. (ANTARES Collaboration) Search for dark matter towards the Galactic Centre with 11 years of ANTARES data Phys. Lett. B 2020, 805135439.

40. Gozzini R. et al. In Proceedings of the 36th International Cosmic Ray Conference (ICRC2019), Madison-WI, USA, 2019; PoS(ICRC2019) 536.

41. Aartsen, M.G. et al. (IceCube Collaboration), Search for neutrinos from dark matter self-annihilations in the center of the Milky Way with 3 years of IceCube/DeepCore, Eur. Phys. J. C 2017 77, no.9, 627

42. Abdallah, H. et al. (HESS collaboration) Search for Dark Matter Annihilations towards the Inner Galactic Halo from 10 Years of Observations with H.E.S.S., Phys. Rev. Lett. 2016 117, no.11, 111301

43. Archambault S. et al. (VERITAS Collaboration), Dark matter constraints from a joint analysis of dwarf Spheroidal galaxy observations with VERITAS, Phys. Rev. D 2017 95, no.8, 082001

44. Ahnen M.L. et al. (MAGIC and Fermi-LAT Collaborations)], Limits to Dark Matter Annihilation Cross-Section from a Combined Analysis of MAGIC and Fermi-LAT Observations of Dwarf Satellite Galaxies, JCAP, 201602 , 039.

45. Albert, A. et al. (ANTARES and IceCube collaborations), Combined search for neutrinos from dark matter self-annihilation in the Galactic Center with ANTARES and IceCube. Phys. Rev. D 2020 102, 082002.

46. Gozzini, S.R. et al. Sensitivity of the ANTARES neutrino telescope for secluded dark matter searches. In Proceedings of the 36th International Cosmic Ray Conference (ICRC2019), Madison-WI, U.S.A., 2019; PoS(ICRC2019) 519 pp. 519.

47. Aartsen, M.G. et al. (IceCube Collaboration), Search for neutrinos from decaying dark matter with IceCube Eur. Phys. J. C 2018 78 no. 10,831 .

48. Albert, A. et al. (HAWC Collaboration), Search for dark matter gamma-ray emission from the Andromeda Galaxy with the High-Altitude Water Cherenkov Observatory, JCAP 201806 043. [Erratum: JCAP 2019 04, E01].

49. Albert, A. et al. (HAWC Collaboration), Dark Matter Limits from Dwarf Spheroidal Galaxies with the HAWC Gamma-Ray Observatory Astrophys. J. 2018853 no. 2, 154.

50. Abeysekara A.U. et al. (HAWC Collaboration), A search for dark matter in the Galactic halo with HAWC JCAP 201802049.

51. Ackermann M. et al. (Fermi-LAT Collaboration) Constraints on the Galactic halo dark matter from Fermi-LAT diffuse measurements Astrophys. J. 201276191.

52. Jeong M. et al. A Search for Neutrinos from Decaying Dark Matter in Galaxy Clusters and Galaxies with IceCube. In Proceedings of the 37th International Cosmic Ray Conference (ICRC2021), Berlin, Germany, 2021; PoS(ICRC2021) 506, pp. 506.

53. Bell N.F., Dola, M.J., Robles S. Searching for Dark Matter in the Sun using Hyper-Kamiokande, arXiv:2107.04216v1.

54. Avrorin et al., Neutrino signal at Baikal from dark matter in the Galactic Center, In Proc of The International Workshop on Prospects of Particle Physics: Neutrino Physics and Astrophysics, 2015, Valday, Russia. 